\title{
Una experiencia presencial de Introducción a la Economía de la Empresa que devino remota
}

\section{A face-to-face experience of Introduction to Business \\ Economics that shifted to a remote one}

JuLIo D. VeCino GRAVEL

ORCID: https://orcid.org/0000-0003-2552-7522

Universidad de Sevilla

Departamento de Administración de Empresas y

Marketing

juliovecino@us.es

DOI: http://dx.doi.org/10.12795/9788447231003.070

Pp.: 1486-1508 


\section{Contexto}

Al comenzar esta experiencia docente todavía al menos la mitad de los estudiantes asistían a clase, la otra mitad se situaba detrás de la plataforma de enseñanza virtual. Esto llevó a plantear la docencia fundamentalmente de manera on-line, solicitando a los estudiantes que estaban en el aula que utilizaron sus teléfonos móviles facilitando que la experiencia fuera lo más unitaria posible. Cuando se empezó a diseñar esta experiencia parecía que lo más adecuado era considerar esta circunstancia y muchas actividades se basaban en búsquedas y recursos disponibles en Internet a los que se accedía de modo individual. Cuando se pasa al modo totalmente remoto, justo antes de iniciar esta experiencia, las actividades se basaron en el trabajo en equipo para evitar que la falta de contacto visual impidiera que las búsquedas en Internet fueran fructíferas. Esto supuso, en contra de lo esperado, un aumento en la dinamicidad de la experiencia.

La asignatura Introducción a la Economía de la Empresa (Organización) pertenece al primer curso del Grado en Marketing e Investigación de Mercados. Los estudiantes de este grado esperan estudiar marketing desde el primer momento y se encuentran con un curso donde predominan asignaturas generales y de base con una alta carga de conceptos nuevos extraños para ellos. Esto supone con frecuencia que la motivación intrínseca brille por su usencia. Por ende, la asignatura tiene fama de presentar especiales dificultades.

Ciclos de Mejora en el Aula (2020). Experiencias de Innovación Docente de la US Esta obra se distribuye con la licencia Creative Commons 


\section{Diseño previo del CIMA}

El ciclo consta de dos mapas de contenidos referidos respectivamente a la gestión de stocks y a la programación de proyectos (Barroso et al., 2012). El primer mapa consta de dos subproblemas y se abarca en su totalidad durante tres sesiones de dos horas, mientras que el segundo, al disponer únicamente de una sesión, solo abarca un subproblema que introduce el concepto general de programación de proyectos (Figuras 1 y 2).

El primer subproblema de gestión de inventarios se refiere a la decisión sobre la cantidad a pedir de un producto y lleva aparejada la frecuencia de los pedidos e, indirectamente, las dimensiones del almacén. El segundo subproblema implica la cuestión temporal, es decir, la antelación con la cual es necesario llevar a cabo el pedido para que el nivel de inventario siempre se mantenga por encima del nivel deseado. Ambos problemas para poder ser abordados necesitan de ciertas hipótesis que deriven en un modelo sencillo y a la vez eficaz. Ambos subproblemas entrañan contenidos conceptuales que permiten explicitar las hipótesis y contenidos procedimentales ya que es un problema práctico que requiere cálculos que permitan, o al menos proporcionen, un marco verosímil para tomar las decisiones adecuadas.

Cada subproblema está rodeado de una pléyade de contenidos conceptuales que soportan las hipótesis y facilitan la comunicación.

Ciclos de Mejora en el Aula (2020). Experiencias de Innovación Docente de la US Esta obra se distribuye con la licencia Creative Commons 

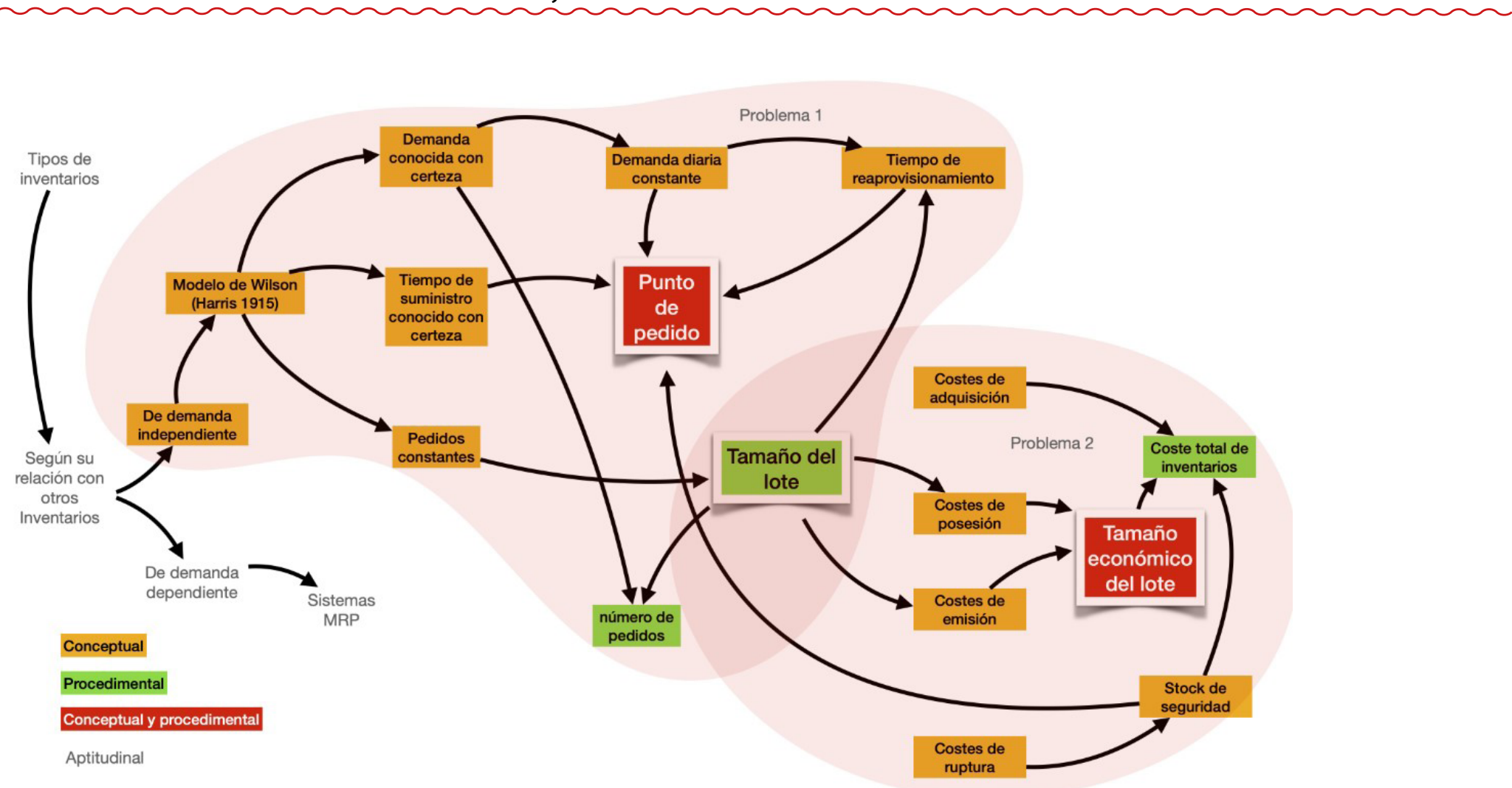

Figura 1. Mapa de contenidos y problema 1

Ciclos de Mejora en el Aula (2020). Experiencias de Innovación Docente de la US (c) (i) $\odot$ Esta obra se distribuye con la licencia Creative Commons 
Se espera que se comprendan las hipótesis del modelo de Wilson para la gestión de inventarios y a su vez que se comprenda la utilidad que tienen los modelos para la gestión de empresas. Nuestra experiencia indica que los estudiantes suelen inclinarse por aprender la fórmula por la que se determina cuál es el lote económico, y reducen a esa fórmula todo el modelo, cuando lo más importante no es precisamente la fórmula sino las hipótesis que efectivamente la sustentan. En orden a conseguir esto, dicha fórmula se deja como material que han de estudiar pero que no es discutido en clase. Con frecuencia los estudiantes que llegan al primer curso enfocan los problemas como la necesidad de encajar piezas en huecos con la misma forma, pero no están acostumbrados a entender los motivos por los cuales las piezas y los huecos son así.

Ciclos de Mejora en el Aula (2020). Experiencias de Innovación Docente de la US Esta obra se distribuye con la licencia Creative Commons 


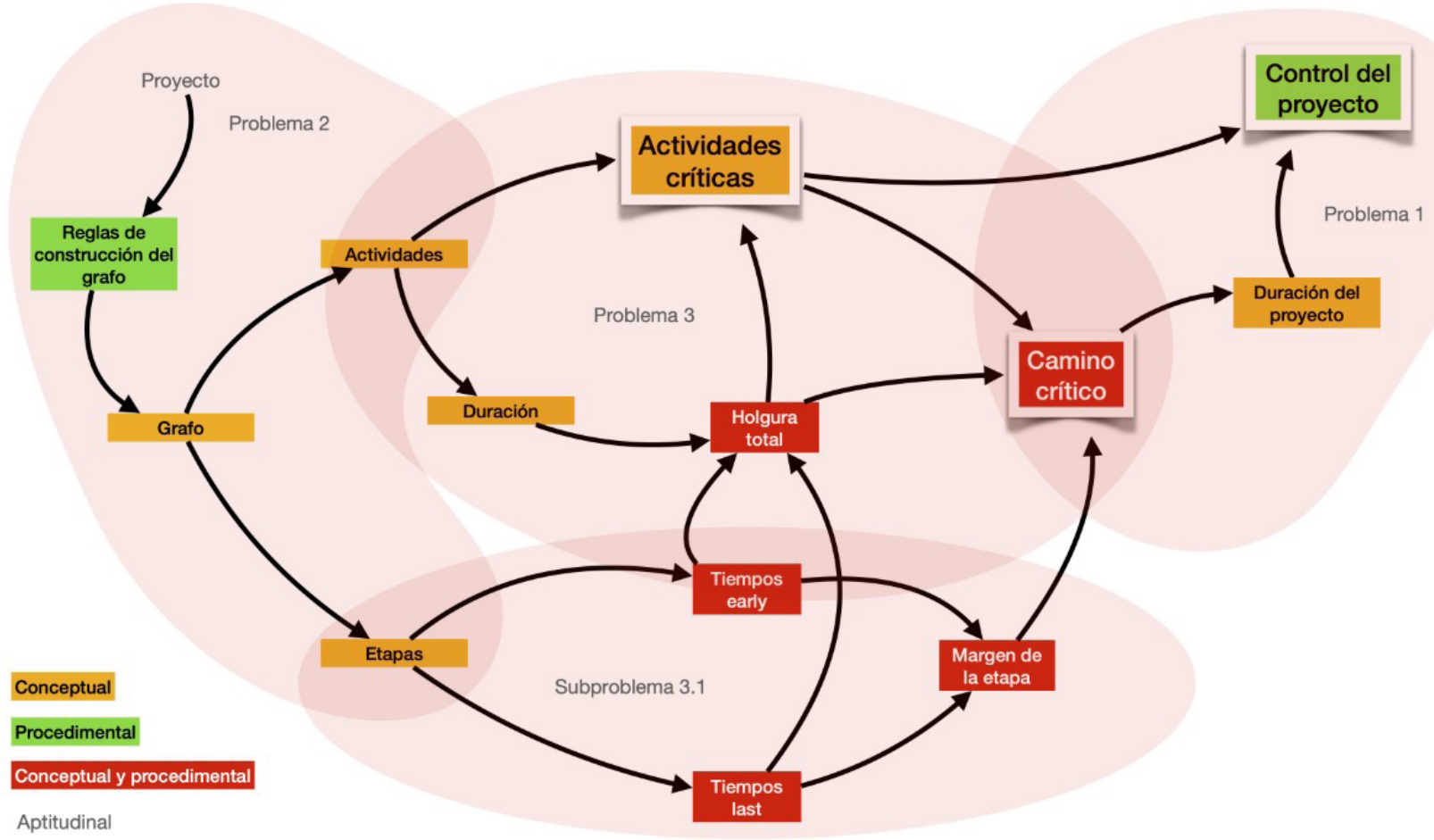

Figura 2. Mapa de contenidos y problema 2

Ciclos de Mejora en el Aula (2020). Experiencias de Innovación Docente de la US cC (7) $\Theta$ Esta obra se distribuye con la licencia Creative Commons (c) Reconocimiento-NoComercial-SinObraDerivada Internacional (CC BY-NC-ND 4.0.) 
Respecto a la gestión de proyectos, como se ha dicho, solo se aborda el primero de los tres subproblemas, aquel que tiene mayor importancia conceptual y soporta todos los elementos constituyentes de los otros dos que tienen una mayor carga procedimental y de los que se dispone en internet de numerosísimos ejemplos y herramientas para llevar a cabo los cálculos necesarios.

En segundo lugar, se espera que entiendan la necesidad de programar actividades cuando los proyectos sean complejos e intervienen múltiples actores. Aquí es importante comprender los conceptos de actividad como consumidora de tiempo y el de precedencia entre actividades. Uno de los resultados que se esperan con mayor interés es el referente a la duración del proyecto cuando tiene varias secuencias de actividades que no se presentan como alternativas si no como concurrentes. Habitualmente, los estudiantes están acostumbrados a considerar secuencias de actividades únicas donde cada actividad va seguida únicamente por otra actividad. Vuelve a ser secundario el aprendizaje del modo en que se calculan cada uno de los elementos necesarios para aprobar proyectos.

Para alcanzar esta meta, en el modelo metodológico utilizado se plantean discusiones en equipos de cuatro o cinco estudiantes formados de modo aleatorio, con el objeto de que, compartiendo información y sin estar condicionados por el profesor, sean capaces de elaborar razonamientos a partir de la pregunta que se les plantea. La misión del profesor consiste en elaborar preguntas relevantes que faciliten el razonamiento a los estudiantes y a completar, si fuera necesario, con información que no han considerado en su trabajo en grupo.

Ciclos de Mejora en el Aula (2020). Experiencias de Innovación Docente de la US Esta obra se distribuye con la licencia Creative Commons 
Cada sesión comienza con un cuestionario referido a los contenidos de la sesión anterior y una posterior ronda de preguntas y resolución de dudas (CX). Esta fase sirve primordialmente para romper el hielo y facilitar que los estudiantes entren en la dinámica de la sesión. En segundo lugar, se plantea una pregunta $(P)$ que es escrita en el chat de la plataforma de enseñanza virtual para que los estudiantes la tengan disponible a lo largo de la siguiente actividad, en la que grupos de cinco estudiantes, seleccionados aleatoriamente con la herramienta de la plataforma, procuran darle respuesta. El profesor lee la pregunta y anima a los estudiantes a que indiquen si han comprendido la tarea que han de realizar. En tercer lugar, los grupos disponen de 20 minutos para resolver la cuestión que se le ha planteado, para posteriormente cada equipo verter en el chat las conclusiones alcanzadas (IA). A partir de ahí, el profesor realiza un comentario general (M) procurando, no solo incluir todo aquello generado por los grupos, sino también proporcionando cierta estructura a las respuestas y completando algún asunto que se ha desvelado más oscuro. Por último, aporta el modo habitual de abordar el problema (T).

La secuencia: pregunta, trabajo en grupo, actividad de contraste y presentación de la respuesta comúnmente admitida en la profesión se repite dos veces en cada sesión. Al final se proporciona un ejercicio (EJ) a realizar fuera de la clase y que será objeto del cuestionario inicial de la sesión siguiente. En dicho cuestionario se suele indicar alguna pregunta que provoque la curiosidad de los estudiantes al no haber sido tratada explícitamente en la sesión o encontrarse situada un poco más adelante de los contenidos abordados hasta el momento. Esto proporciona continuidad a las sesiones sugiriendo que el trabajo del aula continua en casa.

Ciclos de Mejora en el Aula (2020). Experiencias de Innovación Docente de la US Esta obra se distribuye con la licencia Creative Commons 


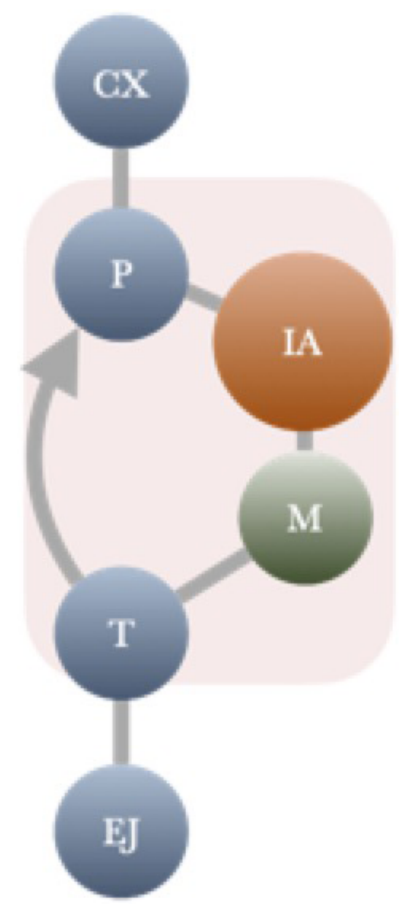

Figura 3. Modelo metodológico

A continuación, se presentan las secuencias de actividades llevadas a cabo. Se incluye, a título de ejemplo, la primera sesión completa y las siguientes preguntas que fueron realizadas.

Tabla 1. Secuencia de actividades completa de la sesión 1

\begin{tabular}{|c|c|c|c|}
\hline CX1 & $\begin{array}{l}\text { Cumplimentación del cuestionario correspondiente } \\
\text { al día anterior, resolución de dudas y lista de clase }\end{array}$ & Sesión 1 & $30^{\prime}$ \\
\hline \multicolumn{4}{|c|}{$\begin{array}{l}\text { Mientras el profesor hace los preparativos para comenzar la clase los } \\
\text { asistentes son invitados a realizar el cuestionario. Cuando el profesor acaba } \\
\text { los preparativos, y mientras los estudiantes terminan el cuestionario, pasa } \\
\text { lista aclarando que no es para controlar la asistencia sino con la intención } \\
\text { de conocer a cada uno personalmente. } \\
\text { Se proporciona a los estudiantes un cuestionario sencillo con únicamente } \\
\text { dos alternativas para mantener la continuidad entre las sesiones y facilitar } \\
\text { el repaso de lo anterior. }\end{array}$} \\
\hline P1 & Hemos planificado y ¿ahora que? & Sesión 1 & $5^{\prime}$ \\
\hline
\end{tabular}

Ciclos de Mejora en el Aula (2020). Experiencias de Innovación Docente de la US Esta obra se distribuye con la licencia Creative Commons Reconocimiento-NoComercial-SinObraDerivada 4.0 Internacional (CC BY-NC-ND 4.0.) 
Ya hemos realizado la planificación y ahora hemos de gestionar los inventarios.

A partir de la planificación estimamos que vamos a necesitar este año unas 54.000 unidades de un consumible que se necesita para hacer soldadura. Hemos de decidir sobre su almacenamiento considerando que es un inventario de demanda independiente ya que depende del uso de la maquinaria pero no de ningún producto específico.

\begin{tabular}{l|l|l|l|}
\hline IA1 & ¿Almacenamos todo lo necesario para el año? & Sesión 1 & $20^{\prime}$
\end{tabular}

Se le plantea a los estudiantes que, por grupos, reflexionen sobre las ventajas e inconvenientes que puede suponer almacenar la cantidad necesaria para todo el año de ese consumible.

M1

Sesión 1

$10^{\prime}$

Se presenta a los estudiantes un gráfico con el modelo de Wilson y las variables relevantes.

T1

Sesión 1 '

A partir de sus intervenciones se indica cual es la perspectiva de la gestión de inventarios para entender cuales son los aspectos relevantes de la materia.

P2

¿Cuales son los costes asociados al almacenamiento?

Sesión 1 5'

Se indica que como se ha podido ver en la pregunta anterior hay varias fuentes de costes en los inventarios.

IA2

¿De que dependen los costes de gestión de los inventarios?

Sesión 1 20'

Se solicita a los estudiantes que reflexionen por grupos sobre los tipos de costes que suponen los inventarios y elaboren soluciones a que tamaño de lote es mejor.

M2

Sesión 1 10'

Se presenta la curva de costes de posesión y de emisión de inventarios para que la contrasten con sus soluciones.

T2

Sesión 1 5'

El profesor explica el cálculo del lote económico.

Ciclos de Mejora en el Aula (2020). Experiencias de Innovación Docente de la US Esta obra se distribuye con la licencia Creative Commons Reconocimiento-NoComercial-SinObraDerivada 4.0 Internacional (CC BY-NC-ND 4.0.) 
Tabla 2. Secuencia de actividades resumida de las sesiones 2,3 y 4

\begin{tabular}{|c|c|c|c|}
\hline P3 & Cálculos básicos en el modelo de Wilson & Sesión 2 & $\mathbf{5}^{\prime}$ \\
\hline \multicolumn{4}{|c|}{$\begin{array}{l}\text { Si la demanda anual es de } 1.260 \text { productos y el tamaño de lote es } 63 \text {, ¿cuál } \\
\text { es el número de pedidos? ¿y el tiempo de reaprovisionamiento? } \\
\text { Si la demanda diaria es de } 5 \text { unidades y el tiempo de reaprovisionamiento } \\
\text { es de } 30, \text { ¿cuál es el tamaño del lote? ¿y el número de pedidos? } \\
\text { Si el tamaño del lote es } 100 \text { y el tiempo de reaprovisionamiento es } 20 \text {, ¿cual } \\
\text { es la demanda diaria? ¿y la demanda? }\end{array}$} \\
\hline P4 & ¿Y si hay stock de seguridad? & Sesión 2 & 5 \\
\hline \multicolumn{4}{|c|}{$\begin{array}{l}\text { Si la demanda anual es de } 1.260 \text { productos y el tamaño de lote es } 63 \text {, ¿Cuál } \\
\text { será el nivel máximo de stocks? ¿Y si el stock de seguridad fuera de } 20 \\
\text { unidades? } \\
\text { Si la demanda diaria es de } 5 \text { unidades y el tiempo de reaprovisionamiento } \\
\text { es de } 30 \text {, ¿Cuál es el stock medio? } \\
\text { Si el tamaño del lote es } 100 \text { y el tiempo de reaprovisionamiento es } 20 \text {, ¿cual } \\
\text { es el número de pedidos? }\end{array}$} \\
\hline P5 & & Sesión 3 & 5 \\
\hline \multicolumn{4}{|c|}{$\begin{array}{l}\text { Visitas el lugar donde se almacena un componente determinado de } \\
\text { la empresa. A la vista de la cantidad de inventario que hay en el, ¿que } \\
\text { información es la mínima que necesitarías para saber cuando hay que hacer } \\
\text { el próximo pedido? }\end{array}$} \\
\hline P6 & & Sesión 3 & $5^{\prime}$ \\
\hline
\end{tabular}

¿Que nivel de inventario es el que nos indica que hemos de hacer el próximo pedido? ¿Cómo se calcula?

\begin{tabular}{|l|l|l|}
\hline $\mathbf{P 7}$ & Sesión $\mathbf{4}$ & $\mathbf{5}^{\prime}$ \\
\hline
\end{tabular}

Si son necesarios 12 días para que llegue un pedido ( $t s=12$ ), calcule el punto de pedido para los ejercicios siguientes:
A) Si la demanda anual es de 1.260 productos y el tamaño de lote es 63
B) Si la demanda diaria es de 5 unidades y el tiempo de reaprovisionamiento es de 30
C) Si el tamaño del lote es 100 y el tiempo de reaprovisionamiento es 20 ¿Habría diferencias si para que llegue un pedido fueran necesarios 20 días?

P8 ¿Cómo programar una celebración?

Sesión 4

5

Ciclos de Mejora en el Aula (2020). Experiencias de Innovación Docente de la US Esta obra se distribuye con la licencia Creative Commons Reconocimiento-NoComercial-SinObraDerivada 4.0 Internacional (CC BY-NC-ND 4.0.) 
Cuatro amigos se están poniendo de acuerdo para organizar la bienvenida a un quinto que viene de estudiar en el extranjero. Uno de ellos lleva en coche a los demás a un centro comercial para que hagan unas compras y después se va al aeropuerto a recoger al que llega. Un segundo va a un supermercado a por material fungible para la recepción en el piso que comparten. Los otros dos buscan elementos que permitan decorar el piso con motivos del país del que viene y al llegar al piso montan la decoración. Con los móviles se aseguran de que el recién llegado no aparezca en el piso antes de que esté todo preparado. Han estimado que tardarán en llegar al centro unos 5 minutos, que ir al aeropuerto, recoger al viajero y llegar al piso serán unos 20 minutos. Las compras en el supermercado duran 10 minutos y las de los elementos decorativos 15. El autobús que va del centro comercial al piso tarda 10 minutos. Decorar el piso lleva 10 minutos más. ¿Cuanto tiempo tardarán en reunirse todos en el piso? ¿Quién será el primero en llamar y a quien para que se de prisa?

Aunque el cuestionario inicial y final se componía de dos preguntas amplias con algunos apartados cuya intención era guiar a los estudiantes en su contestación, aquí solo transcribimos la parte general de cada una.

1. Trabajas para una consultora en el departamento de consultoría de operaciones cuyo objetivo es mejorar los sistemas productivos de las empresas andaluzas y tu jefe te ha indicado que en los próximos meses tu tarea será realizar informes sobre la manera en que manejan sus inventarios distintas empresas. Además, habrás de proporcionar recomendaciones precisas a los responsables de las empresas.

2. Aprovechando tu visita a una empresa que diseña, instala y gestiona centros comerciales te ha pedido ayuda para coordinar la construcción y puesta en marcha de un gran centro comercial a la vista del éxito que está teniendo Lagoh, pero considerando las adaptaciones necesarias a las nuevas circunstancias que van a resultar permanentes al menos durante una época.

Ambas preguntas situaban al estudiante en una situación profesional en las que se les solicitaba su colaboración. Excepto en un caso, todos los estudiantes expresaron

Ciclos de Mejora en el Aula (2020). Experiencias de Innovación Docente de la US Esta obra se distribuye con la licencia Creative Commons 
su punto de vista sobre las cuestiones manifestando, junto a sus ideas, las carencias propias de su nivel de estudios. Solo una persona hizo referencias explícitas a contenidos ya estudiados semanas antes y cuya relación con la pregunta eran remotos. Esto se indica porque excepto esta persona todos proporcionaron las respuestas que le parecían adecuadas sin buscar la aquiescencia del profesor recurriendo a contenidos ya expuestos.

\section{Aplicación del CIMA}

Al ser las sesiones virtuales, temía que al tener la posibilidad de esconderse en el anonimato tras la pantalla los estudiantes no mostraran interés y abandonaran la clase. A esto se unían las dificultades con la tecnología que habíamos tenido desde principio de curso y la tendencia acusada de los estudiantes de primero de abandonar algunas asignaturas tras los primeros compases. Excepción hecha de aquellos estudiantes que ya habían cursado la asignatura en años anteriores, casi todos los estudiantes matriculados han permanecido en clase y en una gran mayoría han participado en las distintas actividades que se han planteado. Incluso, actividades no puntuables a realizar fuera del aula, y del ciclo de mejora han tenido gran acogida.

La mayoría de las sesiones se han realizado desde el despacho de profesor donde hay medios suficientes para lo que se pretende. La utilización de dos monitores permite mayor agilidad al compartir la pantalla, porque es posible que el trabajo del profesor, por ejemplo, ordenando las intervenciones, se pueda ver en vivo. En caso contario durante ese tiempo los estudiantes tendrían que esperar a la tarea del profesor, sin tener conocimiento ni de si lo que hace es de interés, ni de cuanto tiempo falta hasta que vuelva a estar online.

Ciclos de Mejora en el Aula (2020). Experiencias de Innovación Docente de la US Esta obra se distribuye con la licencia Creative Commons 
El tono general fue de trabajo serio, y las posibles salidas de tono fueron inexistentes. Quizá la única estridencia se produjo alguna vez cuando, al deshacer los grupos de trabajo, alguien no era consciente de que el micrófono estaba abierto y se percibía un tono excesivamente coloquial. Los grupos de trabajo han resultado una herramienta valiosísima, aunque en un principio no iban a tener tanto protagonismo como lo han tenido a la postre.

Desde el punto de vista del profesor, la experiencia ha sido muy enriquecedora y sorprendente. El efecto Pigmalión resultó mucho más efectivo de lo que podía esperar y a veces era difícil terminar la sesión porque querían seguir con los asuntos tratados. Además, expresaban sus opiniones con naturalidad sin temor a ser reconducidos. El uso intensivo del trabajo en grupo de los estudiantes lleva a un esfuerzo notable por parte del profesor, que ha de poner todo su empeño en entender que es lo que los estudiantes manifiestan para ordenarlo y hacerlo mas comprensible y formalizado, de modo que todos se puedan beneficiar de las aportaciones de los demás.

Los tiempos previstos en las secuencias de actividades se tornaron muy orientativos ya que las diferencias de interés y velocidad de ejecución estimadas y efectivamente realizadas fueron notables.

El trabajo de preparación de las actividades resultó con frecuencia harto dificultoso al tener que plantear la pregunta o problema oportuno que permitiera que los estudiantes iniciaran el proceso de aprendizaje. Es complicado no solo que provoquen respuestas en el estudiante, sino que además dicha respuesta permita seguir avanzando.

Esta asignatura supone un magnífico banco de pruebas para un enfoque constructivista dado que la mayor 
parte de los contenidos están muy relacionados, o pueden relacionarse con cierta facilidad, con situaciones cotidianas que pueden ser transformadas posteriormente en situaciones profesionales. Este hecho ha supuesto que en más de una ocasión las propuestas sugeridas por los estudiantes hayan sorprendido al profesor no solo por su originalidad sino por su pertinencia y agudeza.

Finalmente, resulta arduo admitir la paradoja de que a los estudiantes les cuesta comprender que hay problemas que han de resolverse y a la vez que es preciso simplificar la realidad mediante modelos para poder abordar los problemas con éxito. De algún modo, piensan que los todos los problemas ya están resueltos y por tanto que ellos son meros espectadores de lo que los docentes llevan a cabo. Junto a esto estiman que los modelos son demasiado sencillos y exigen incluir elementos o relajar hipótesis que haga los problemas más reales. Es como si estimaran que, ya que como ellos son espectadores, los profesores como representantes del mundo adulto hemos de hacer ejercicios imposibles.

El aprendizaje ha sido diverso respecto a los dos problemas planteados. En el caso del primer problema, al que se han dedicado tres sesiones, el aprendizaje ha sido más intenso, mientras que en el segundo ha sido más discreto. Se presentan a continuación las dos escaleras de aprendizaje diseñadas correspondientes a los dos problemas.

El cuestionario inicial fue respondido por dieciséis personas y el final únicamente por once y aquí se presentan únicamente los resultados inicial y final de estas once. Algún estudiante ha manifestado que no contestaba el segundo cuestionario por tener actividades que incidian en mayor medida en la calificación de otras asignaturas y se sentía escaso de tiempo para acometer todas las exigencias de las distintas asignaturas.

Ciclos de Mejora en el Aula (2020). Experiencias de Innovación Docente de la US Esta obra se distribuye con la licencia Creative Commons 
El cuestionario consta de dos preguntas que después se desglosan en otras tres, en el primer caso, y en cuatro en el segundo. Como el objeto de las preguntas de desglose era facilitar el razonamiento de los estudiantes y su reflexión, se ha considerado oportuno evaluar las preguntas en bloque sin distinguir cada uno de los apartados. Con esto no se pierde generalidad ni a priori ni a posteriori porque de su lectura se desprende que la tónica de todos los apartados de cada pregunta es análoga.

En el cuestionario inicial, en ambas preguntas, he detectado cuatro niveles de respuesta de progresiva complejidad (Porlán, 2017), siendo el quinto el que espero obtener en el cuestionario final (Figura 3). Estos niveles o escalones los he denominado igual porque es posible hacerlo ya que la materia es de gestión de empresas, y ellos son estudiantes de marketing.

En el primer escalón están aquellos estudiantes que, en sus repuestas, divagan o expresan generalidades que podrían responder a casi cualquier pregunta. Estimo que el obstáculo que presentan es el miedo a equivocarse, dado el contexto académico en que se les pregunta. En la segunda pregunta no hay nadie en este primer escalón más débil, porque al estar incluidas de modo velado las circunstancias de la pandemia, y al haber comentado ya en clase anteriormente la empresa que figura en el ejemplo, los alumnos se han debido sentir más seguros.

En el segundo escalón el obstáculo se produce porque intentan hacer ver que son de marketing y por tanto quedan eclipsados los aspectos de gestión que les resultan menos atractivos. Esperan que se les reconozca como profesionales del marketing.

En el tercer escalón están la gran mayoría haciendo referencias a aspectos de gestión de modo general y con 
un espectro de acciones más amplio que en el escalón anterior. Para pasar al cuarto escalón han de superar de nuevo la aversión al fracaso y concretar el modo de llevar a la práctica sus ideas. La incapacidad de concretar en planes de acción suele ser una carencia en los estudiantes universitarios.

En el quinto escalón no hay ningún representante, ya que parece que no esperan que sea necesario aplicar ninguna técnica concreta al ver que no hay datos en las preguntas y su hábito es de responder a los números con números y a las letras con letras.

Respecto a la evolución de la primera escalera en el cuestionario final, algunos estudiantes han permanecido en el primer escalón debido probablemente a repetir el cuestionario anterior por no haber seguido el ciclo o por la presión recibida desde otras asignaturas. La gestión del tiempo es una asignatura pendiente como competencia transversal imprescindible para un buen rendimiento académico y profesional.

Excepción hecha de los que han permanecido en el escalón inferior todos los demás han evolucionado alcanzando los dos escalones superiores, reflejando un claro aprendizaje. Algunos han subido un escalón, pero otros han subido dos de una tacada alcanzando la cima no alcanzada por nadie en el cuestionario inicial.

Es notable cómo de un cuestionario a otro han desaparecido los prejuicios que parecía haber respecto a la gestión, ya que todos aquellos que hacían hincapié en cuestiones comerciales han superado ese obstáculo. También es de reseñar que más de la mitad de los que han respondido ambos cuestionarios han alcanzado el último escalón.

Ciclos de Mejora en el Aula (2020). Experiencias de Innovación Docente de la US Esta obra se distribuye con la licencia Creative Commons 


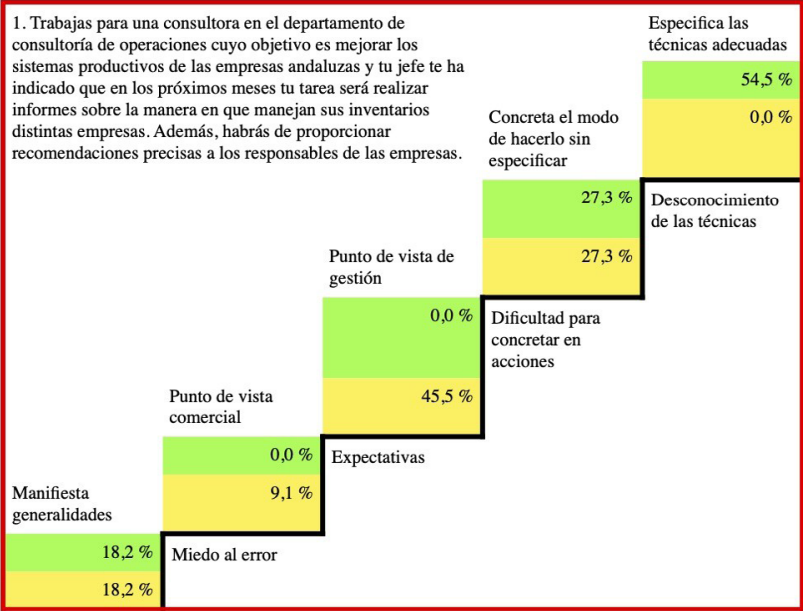

Figura 4. Escalera de aprendizaje de la pregunta 1

En el segundo caso los resultados son menos claros, quizá porque la dedicación al problema ha sido más reducida y porque el cuestionario estaba demasiado cerca en el tiempo de la última sesión. Quizá también no ha transcurrido tiempo suficiente para que se pueda apreciar la diferencia entre los escalones tercero y cuarto. Además, en ese caso, quizá el contexto de la pregunta que se planteó como actividad en clase era tan distinto - un grupo de amigosque no se ha asociado a la pregunta del cuestionario.

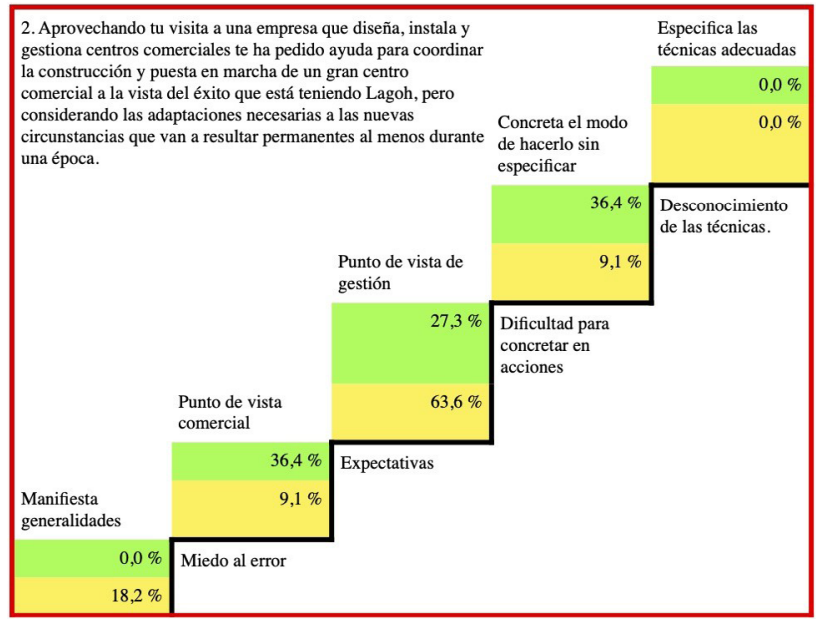

Figura 5. Escalera de aprendizaje de la pregunta 2

Ciclos de Mejora en el Aula (2020). Experiencias de Innovación Docente de la US Esta obra se distribuye con la licencia Creative Commons 
Es de destacar que en los cuestionarios finales aquellos contenidos que no han sido objeto de las sesiones, sino que fueron recomendados para estudio personal, no han aflorado en absoluto, manteniéndose aquello que escribieron en el cuestionario inicial. Aunque esos contenidos no eran centrales, esperaba que fueran atendidos. Es preciso, por tanto, incorporar de alguna manera elementos que lleven a los estudiantes a ampliar su visión fuera de la clase.

A continuación, se detalla un cuadro con la evolución de cada estudiante.

Tabla 3. Cuadro de evolución de cada estudiante

\begin{tabular}{|c|c|c|c|c|c|c|c|}
\hline Estudiante & 1 inicial & 1 final & 1 aumento & 2 inicial & 2 final & 2 aumento & aumento \\
\hline 1 & 2 & 4 & 2 & 1 & 2 & 1 & 3 \\
\hline 2 & 3 & 5 & 2 & 3 & 3 & 0 & 2 \\
\hline 3 & 3 & 5 & 2 & 3 & 3 & 0 & 2 \\
\hline 4 & 2 & 4 & 2 & 1 & 2 & 1 & 3 \\
\hline 5 & 2 & 1 & -1 & 3 & 2 & -1 & -2 \\
\hline 6 & 3 & 5 & 2 & 3 & 3 & 0 & 2 \\
\hline 7 & 2 & 1 & -1 & 4 & 2 & -2 & -3 \\
\hline 8 & 4 & 4 & 0 & 3 & 4 & 1 & 1 \\
\hline 9 & 4 & 5 & 1 & 3 & 4 & 1 & 2 \\
\hline 10 & 4 & 5 & 1 & 2 & 4 & 2 & 3 \\
\hline 11 & 4 & 5 & 1 & 3 & 4 & 1 & 2 \\
\hline & & & & & & & \\
\hline
\end{tabular}

Como se ha dicho, contestaron el cuestionario inicial y final once personas de las dieciséis que contestaron el inicial.

Se puede comprobar en el cuadro que los resultados son mejores en la primera pregunta que en la segunda aún partiendo de una situación análoga. Esto avala el hecho de que la primera pregunta se refiere a tres sesiones y la segunda a exclusivamente a una y que ha podido recoger

Ciclos de Mejora en el Aula (2020). Experiencias de Innovación Docente de la US Esta obra se distribuye con la licencia Creative Commons 
cierta presión debida al avance del curso y a la irrupción de los parciales de otras asignaturas en esa semana. En el cuadro se observa que todos los estudiantes progresan excepto dos que además retroceden en ambas preguntas.

\section{Evaluación del CIMA}

Los resultados del ciclo han sido muy satisfactorios, superando todas las expectativas. Casi todo lo aprendido en el es susceptible de ser incorporado a las clases. Antes de aplicarlo, y gracias al ciclo de mejora anterior, ya se incorporó el que podemos denominar cuestionario de continuidad. Este surgió de un modo espontáneo cuando las clases eran aún presenciales y se hacía muy lento el inicio de la clase por dificultades tecnológicas. Es frustrante tanto para el profesor como para los estudiantes el estar en clase durante muchos minutos esperando que el ordenador arranque, que aparezca la hoja de firmas y que se carguen las presentaciones. Lo más sencillo era que mientras los estudiantes hicieran un cuestionario el profesor pasara lista, no con ánimo de controlar la asistencia, sino con la intención de conocer mejor a los enmascarados a los que había de dirigirse. Si no os veo las caras al menos conozco vuestro nombre.

El mapa de contenido y la secuencia de actividades han de estar mucho mejor integrados de modo que siempre se sepa el momento del itinerario. Numerar los contenidos puede ayudar al despliegue en la clase, ya que lo queramos o no el tiempo solo va hacia adelante y además es escaso. Eso no significa que no pueda haber itinerarios alternativos, sino que es necesario preverlos.

Cada una de las preguntas que se plantean han de estar probadas y redactadas de modo claro sin ambigüedades. Es preciso dedicar mucho tiempo a diseñarlas, ya que una pregunta mal diseñada aparte de ser poco útil 
desconcierta sobremanera a los estudiantes. Es importante asegurarse que la pregunta se entiende bien y dedicar el tiempo que sea necesario a precisar su sentido para que el trabajo de los estudiantes sea más efectivo.

Sería mejor, aunque las actividades tengan cierta homogeneidad para facilitar rutinas de aprendizaje, añadir ocasionalmente actividades diferentes que dinamicen la clase y faciliten retomar el curso a aquellos que lo habían perdido.

Aprovechar la tecnología para incrementar las posibilidades de autoevaluación sin exigir al profesor corregir muchas actividades parece una vía prometedora en grupos grandes.

Es necesario hacer una planificación donde el tiempo previsto para cada sesión sea inferior al disponible y, a su vez, contar con actividades adicionales para proporcionar suficiente flexibilidad, de modo que ni se excluyan contenidos ni se acorte el tiempo por falta de previsión, con el consiguiente desinterés de los estudiantes que pueden interpretar que no es importante el tiempo de clase en perjuicio de su aprendizaje.

Es claro que la labor docente ha de estar centrada en el estudiante porque los conocimientos están disponibles y la tarea del profesor ha de ser la de guía y catalizador en el aprendizaje. Para ello es necesario conocer donde están los estudiantes para ir hacia ellos: la iniciativa ha de ser del profesor a causa de su mayor conciencia de la necesidad de aprender. También el profesor ha de hacer el esfuerzo de adaptar los contenidos a su audiencia formulando preguntas relevantes en ambas orillas: la del estudiante y la del conocimiento a adquirir. 
En las circunstancias actuales de la asignatura no parece posible modificar el sistema de calificación, pero si es posible hacer esfuerzos para que la evaluación que se lleva a cabo sobre las actividades del curso vea su reflejo en la calificación. El esfuerzo del profesor ha de ir dirigido a que las actividades no solo faciliten el aprendizaje de los estudiantes, sino que también les proporcione las herramientas necesarias para hacer frente con éxito a los exámenes.

Una conclusión obtenida es que una gran cantidad de estudiantes por grupo puede dificultar la tarea del profesor, pero eso no debe llevarle a dejar de buscar nuevos caminos que permitan mejorar el aprendizaje de los universitarios.

Ciclos de Mejora en el Aula (2020). Experiencias de Innovación Docente de la US Esta obra se distribuye con la licencia Creative Commons 
Palabras clave: Introducción a la Economía de la Empresa (Organización); Docencia Universitaria, Experimentación Docente Universitaria, Trabajo en Equipo

Keywords: Introduction to Business Economics (Organization); University Teaching; University Teaching Experimentation, Teamwork

\section{Referencias bibliográficas}

Barroso, C., Casillas, J., Villegas, M., Vecino, J., y Calvo de Mora, A. (2012). Economía de la Empresa. Madrid: Pirámide.

Porlán, R. (2017). Enseñanza Universitaria. Cómo mejorarla. Madrid: Ediciones Morata.

Ciclos de Mejora en el Aula (2020). Experiencias de Innovación Docente de la US Esta obra se distribuye con la licencia Creative Commons 